\title{
Surveillance sentinelle du risque de la maladie de Lyme au Canada, 2019 : résultats de la première année du Réseau sentinelle canadien de surveillance de la maladie de Lyme (ReSCaL)
}

\author{
Camille Guillot ${ }^{1,2 *}$, Jackie Badcock ${ }^{3}$, Katie Clow ${ }^{4}$, Jennifer Cram $^{5}$, Shaun Dergousoff ${ }^{6}$, \\ Antonia Dibernardo ${ }^{7}$, Michelle Evason 6,8 , Erin Fraser ${ }^{9,10}$, Eleni Galanis ${ }^{11}$, Salima Gasmi ${ }^{12}$, \\ Greg J German ${ }^{13}$, Douglas T Howse ${ }^{14}$, Claire Jardine ${ }^{6}$, Emily Jenkins ${ }^{15}$, Jules Koffi ${ }^{13}$, \\ Manisha Kulkarni ${ }^{16}$, L Robbin Lindsay ${ }^{8}$, Genevieve Lumsden 6 , Roman McKay ${ }^{17}$, Muhammad \\ Morshed ${ }^{12}$, Douglas Munn ${ }^{18}$, Mark Nelder ${ }^{19}$, Joe Nocera ${ }^{19}$, Marion Ripoche ${ }^{20}$, Kateryn Rochon ${ }^{21}$, \\ Curtis Russell ${ }^{20}$, Andreea Slatculescu ${ }^{17}$, Benoit Talbot ${ }^{17}$, Karine Thivierge ${ }^{22}$, Maarten Voordouw ${ }^{16}$, \\ Catherine Bouchard ${ }^{1,23}$, Patrick Leighton ${ }^{1}$
}

\section{Résumé}

Contexte : La maladie de Lyme est une zoonose émergente à transmission vectorielle d'importance croissante au Canada en termes de santé publique. Dans le cadre de son mandat, le Canadian Lyme Disease Research Network (CLyDRN) a mis en place en 2019 une initiative de surveillance sentinelle au niveau pancanadien, le Réseau sentinelle canadien de la surveillance de la maladie de Lyme (ReSCaL).

Objectifs : La création d'un réseau de surveillance sentinelle national permettant d'évaluer l'évolution du risque environnemental de la maladie de Lyme en temps réel dans chaque province.

Méthodes : Une approche d'analyse décisionnelle multicritère (ADMC) a été utilisée pour la sélection des régions sentinelles. Dans chaque région sentinelle, un protocole d'échantillonnage systématique par méthode de la flanelle a été réalisé dans des sites d'échantillonnage sélectionnés. Les tiques collectées au cours de ces visites de surveillance active ont été identifiées par espèce, et seulement les tiques lxodes spp. ont été testées pour détecter des agents pathogènes tels que Borrelia burgdorferi, Borrelia miyamotoi, Anaplasma phagocytophilum, Babesia microti et le virus Powassan.

Résultats : En 2019, un total de 567 tiques Ixodes spp. (I. scapularis [ $=550$ ] I. pacificus [ $=10]$; et $l$. angustus [ $n=7]$ ) ont été collectées dans sept provinces : en

Colombie-Britannique, au Manitoba, en Ontario, au Québec, au Nouveau-Brunswick, en Nouvelle-Écosse et à Île-du-Prince-Édouard. Les plus hautes densités moyennes de tiques (nymphes $/ 100 \mathrm{~m}^{2}$ ) ont été observées dans les régions sentinelles de Lunenburg $(0,45)$, Montréal $(0,43)$ et Granby $(0,38)$. Globalement, la prévalence de la Borrelia burgdorferi chez les tiques était de $25,2 \%$ (entre $0 \%$ et $45,0 \%$ ). En Colombie-Britannique, une nymphe $I$. angustus a testé positive pour Babesia microti, une première dans la province. La lignée du virus Powassan a été détectée chez une I. scapularis adulte en Nouvelle-Écosse.

Conclusion : Le ReSCaL représente la première initiative nationale de surveillance active pour les maladies transmises par les tiques au Canada. Grâce à des collaborations multidisciplinaires entre des experts provinciaux, l'année pilote a permis d'établir une référence de base pour le risque de maladie de Lyme au pays, et permettra l'étude des tendances futures.
Cette oeuvre est mise à la disposition selon les termes de la licence internationale Creative Commons Attribution 4.0

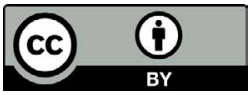

Affiliations

*Voir la liste complète d'affiliations dans l'appendice

${ }^{\star}$ Correspondance : camille.guillot@umontreal.ca 
Citation proposée : Guillot C, Badcock J, Clow K, Cram J, Dergousoff S, Dibernardo A, Evason M, Fraser E, Galanis E, Gasmi S, German GJ, Howse DT, Jardine C, Jenkins E, Koffi J, Kulkarni M, Lindsay LR, Lumsden G, McKay R, Morshed M, Munn D, Nelder M, Nocera J, Ripoche M, Rochon K, Russell C, Slatculescu A, Talbot B, Thivierge K, Voordouw M, Bouchard C, Leighton P. Surveillance sentinelle du risque de la maladie de Lyme au Canada, 2019 : résultats de la première année du Réseau canadien sentinelle de la maladie de Lyme (RCSMLy). Relevé des maladies transmissibles au Canada 2020;46(10):399-407.

https://doi.org/10.14745/ccdr.v46i10a08f

Mots-clés : maladie de Lyme, surveillance sentinelle, tique Ixodes, Borrelia, Canadian Lyme Disease Research Network (CLyDRN)

\section{Introduction}

Au Canada, la maladie de Lyme est une zoonose émergente à transmission vectorielle d'importance croissante en termes de santé publique (1). La maladie de Lyme est causée par la bactérie Borrelia burgdorferi et est principalement transmise aux les humains par la tique à pattes noires (Ixodes scapularis) dans le centre et l'est du Canada et par la tique à pattes noires de l'ouest (Ixodes pacificus) en Colombie-Britannique. Depuis que la maladie de Lyme est devenue une maladie à déclaration obligatoire nationalement en 2009, le nombre de cas confirmés annuellement est passé de 144 à plus de 2000 en 2017 (2,3).

En réponse au risque croissant de la maladie de Lyme au sein de la population canadienne et aux lacunes actuelles dans les connaissances, les Instituts de recherche en santé du Canada (IRSC) ont financé la création d'un réseau national de recherche sur la maladie de Lyme (4). Lancé en 2018, le Canadian Lyme Disease Research Network (CLyDRN) est une initiative multidisciplinaire qui rassemble des patients, des médecins, des spécialistes des sciences sociales, des vétérinaires et des chercheurs universitaires et gouvernementaux dans le cadre d'une approche centrée sur le patient et visant à améliorer le diagnostic, la surveillance, la prévention et le traitement de la maladie de Lyme au Canada. L'un des principaux objectifs du réseau est de mieux comprendre le risque de maladie de Lyme dans tout le pays et son évolution. Ainsi, l'une des premières actions du réseau a été de mettre en place une structure de surveillance pancanadienne pour recueillir des données comparables sur les risques environnementaux dans tout le pays.

Une considération importante dans la planification de la surveillance est que le risque de maladie de Lyme n'est pas uniforme dans tout le pays (5). Cela est dû en grande partie aux différences régionales entre les espèces de tiques et l'environnement (6-9) et au schéma irrégulier de l'expansion dans l'espace des populations d'Ixodes scapularis au Canada (10). Les différences régionales dans le statut socio-économique des canadiens sont également susceptibles $d$ 'influencer la manière dont le risque environnemental affecte l'incidence régionale des cas de maladie de Lyme (11).

Bien que des efforts considérables aient été investis dans l'évaluation du risque de maladie de Lyme pour les canadiens, la surveillance reste hétérogène à travers le pays. La surveillance passive, c'est-à-dire la soumission de tiques collectées sur des humains ou des animaux, fournit des informations importantes à propos de ce risque $(12,13)$, mais ne peut être maintenue de manière uniforme dans tout le pays en raison de ressources limitées. La surveillance active, c'est-à-dire la collecte de tiques dans l'environnement grâce à l'échantillonnage par méthode de la flanelle ou par la capture de rongeurs, est une activité qui nécessite des ressources intensives et est dirigé au niveau provincial, en fonction des objectifs spécifiques de la région, de leurs protocoles d'échantillonnage et des fonds disponibles.

Ce rapport présente les premiers résultats du Réseau sentinelle canadien de surveillance de la maladie de Lyme (ReSCaL), un nouveau réseau pancanadien de surveillance de la maladie de Lyme établi par CLyDRN en 2019. Au cours de cette année pilote, une surveillance active standardisée des tiques dans l'environnement a été effectuée à travers le Canada, en utilisant une approche de surveillance sentinelle. La surveillance sentinelle permet de concentrer les efforts de surveillance dans des régions sentinelles sélectionnées, et de fournir une mesure comparable du risque environnemental de la maladie de Lyme et d'autres maladies transmises par les tiques dans tout le pays, ainsi que de l'information approfondie qui complémentent les activités de surveillance fédérales et provinciales en cours.

\section{Objectifs}

Les objectifs du ReSCaL sont de surveiller annuellement les régions sentinelles de chaque province canadienne pour : 1) fournir le premier portrait national normalisé, en temps réel, de l'évolution du risque environnemental de la maladie de Lyme au Canada et 2) soutenir la recherche sur les variations régionales du risque et ses déterminants.

\section{Méthodes}

\section{Sélection des régions sentinelles}

Les régions sentinelles ont été sélectionnées par le groupe de travail du ReSCaL, un groupe d'experts universitaires et de santé publique en surveillance des maladies transmises par les tiques. Les régions sentinelles ont été définies géographiquement comme les zones situées dans un rayon de $25 \mathrm{~km}$ autour du centre géographique des municipalités focales sélectionnées. Le 
groupe de travail a utilisé une approche d'analyse décisionnelle multicritères (ADMC) pour prioriser entre une et quatre régions sentinelles par province, avec l'objectif d'inclure des régions supplémentaires au fil du temps (14).

Les critères de sélection comprenaient l'évidence d'émergence de la maladie de Lyme basées sur les données de surveillance passive existantes (nombre de soumissions de tiques Ixodes spp./100 000 personnes) (10); la densité de la population humaine couverte par le réseau; et des critères logistiques associés à l'échantillonnage et à la disponibilité d'un environnement propice aux tiques lxodes spp., tels que la présence de forêts feuillues ou mixtes. Aucune région sentinelle n'a été établie au Yukon, dans les Territoires du Nord-Ouest, au Nunavut ou dans la partie continentale de Terre-Neuve-et-Labrador parce que les conditions environnementales actuelles à ces latitudes ne sont pas propices à l'établissement des tiques Ixodes spp. (15).

\section{Collecte des tiques}

Les tiques ont été collectées dans chaque région sentinelle à l'aide d'un protocole d'échantillonnage standardisé par la méthode de la flanelle $(16,17)$. II s'agissait de traîner un morceau de flanelle blanche de $1 \mathrm{~m} \times 1 \mathrm{~m}$ sur $2000 \mathrm{~m}^{2}$ de végétation au sol en transects linéaires, tout en s'arrêtant tous les $25 \mathrm{~m}$ pour ramasser les tiques qui s'étaient accrochées à la flanelle. Plusieurs sites d'échantillonnage ont été sélectionnés dans chaque région sentinelle. Les lieux ont été choisis parce qu'ils offraient un habitat approprié pour les tiques. Les efforts de surveillance ont été intensifiées dans les zones endémiques connues de la maladie de Lyme afin d'obtenir des informations à fine échelle sur la répartition du risque dans ces endroits (tableau 1). Chaque site a été échantillonné une fois au cours de l'été (entre mai et août 2019), en ciblant par région le pic d'activité des nymphes Ixodes spp., le stade le plus important en termes de santé publique (18-20). En plus de la collecte de tiques, nous avons recueilli des données sur la profondeur de la litière, la canopée et l'humidité du sol à chaque site d'échantillonnage et avons noté la température ambiante et les conditions météorologiques pendant la collecte afin de tenir compte des effets possibles de ces variables sur la collecte de tiques.

\section{Analyses de laboratoire}

Toutes les tiques collectées par la méthode de la flanelle ont été identifiées à l'espèce, mais seules les tiques $I$. scapularis $(n=550)$, l. pacificus $(n=10)$ et $l$. angustus $(n=7)$ ont été testées pour la présence d'agents pathogènes, car elles sont des vecteurs connus de $B$. burgdorferi et d'autres agents pathogènes. La présence d'Anaplasma phagocytophilum, de Babesia microti, de B. burgdorferi, de Borrelia miyamotoi et du virus Powassan a été analysé chez les tiques individuellement par réaction en chaîne de la polymérase $(P C R)$ en temps réel ou par transcriptase inverse-PCR, avec de légères modifications des méthodes décrites précédemment (21). En bref, les acides nucléiques ont été extraits des tiques à l'aide des trousses QIAGEN RNeasy 96 (QIAGEN Inc., Mississauga, Ontario, Canada). Les extraits contenaient à la fois de l'ARN et de l'ADN, et ont été soumis à un test de dépistage de tous les agents pathogènes énumérés ci-dessus. Les modifications apportées aux algorithmes de test comprenaient l'utilisation d'un test de

Tableau 1 : Densité des nymphes Ixodes spp. collectées dans tous les sites d'échantillonnage de chaque région sentinelle du Réseau sentinelle canadien pour la surveillance de la maladie de Lyme, 2019

\begin{tabular}{|c|c|c|c|c|c|}
\hline \multirow{2}{*}{ Région sentinelle } & \multirow{2}{*}{$\begin{array}{l}\text { Nombre de } \\
\text { sites visités }(n)\end{array}$} & \multicolumn{3}{|c|}{ Densité (nombre de nymphes / $100 \mathrm{~m}^{2}$ ) } & \multirow{2}{*}{ Écart-type } \\
\hline & & Minimum & Maximum & Moyenne & \\
\hline Vancouver, C.-B. & 5 & 0 & 0,10 & 0,04 & 0,04 \\
\hline Lethbridge, Alb. & 3 & 0 & 0 & 0 & 0 \\
\hline Saskatoon, Sask. & 3 & 0 & 0 & 0 & 0 \\
\hline Winnipeg, Man. & 5 & 0 & 0 & 0 & 0 \\
\hline Hamilton, Ont. & 5 & 0 & 0,10 & 0,02 & 0,04 \\
\hline Kingston, Ont. & 15 & 0,05 & 1,15 & 0,27 & 0,38 \\
\hline Ottawa-Gatineau, Ont./Qc & 10 & 0 & 0,40 & $0,06^{a}$ & 0,12 \\
\hline Montréal, Qc & 10 & 0 & 2,90 & 0,43 & 0,91 \\
\hline Granby, Qc & 5 & 0 & 1,15 & 0,38 & 0,48 \\
\hline Sherbrooke, Qc & 5 & 0 & 0 & 0 & 0 \\
\hline Saint John, N.-B. & 5 & 0 & 0,30 & 0,09 & 0,13 \\
\hline Charlottetown, Î.-P.-É. & 5 & 0 & 0,05 & 0,01 & 0,02 \\
\hline Lunenburg, N.-É. & 10 & 0 & 2,45 & 0,45 & 0,74 \\
\hline St. John's, T.-N.-L & 5 & 0 & 0 & 0 & 0 \\
\hline
\end{tabular}

Abréviations : Alb., Alberta; C.-B., Colombie-Britannique; Î.-P.-É., Île-du-Prince-Édouard; Man., Manitoba; N.-B., Nouveau-Brunswick; N.-É., Nouvelle-Écosse; Ont, Ontario; Qc, Québec; Sask.,

Saskatchewan; T.-N.-L., Terre-Neuve-et-Labrador

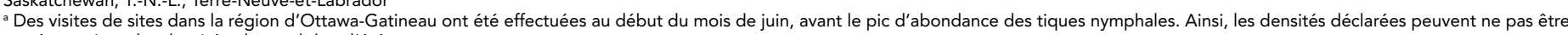
représentatives des densités plus tard dans l'été 
dépistage triplex interne ciblant le gène ARNr 18S des espèces de Babesia, suivi du test CCT-éta en temps réel spécifique à la $B$. microti pour confirmation, ainsi que d'un test duplex (22) pour confirmer la présence de la $B$. burgdorferi et/ou la $B$. miyamotoi.

\section{Analyses statistiques}

Les résultats sont présentés sous forme de statistiques descriptives. Nous avons utilisé un test de Wilcoxon apparié pour comparer la prévalence moyenne de la Borrelia chez les tiques adultes et nymphes. Les analyses ont été effectuées à l'aide de $R$, version 3.6.2 (23)

\section{Résultats}

\section{Régions sentinelles et sites d'échantillonnage} Au total, 96 sites dans 14 régions sentinelles (figure 1) ont été échantillonnés du 22 mai 2019 au 20 août 2019, avec entre trois et 15 sites d'échantillonnage par région (tableau 1).

\section{Tiques des espèces $d^{\prime}$ Ixodes}

Au total, 567 tiques Ixodes spp. ont été collectées dans

10 régions sentinelles de sept provinces : en

Colombie-Britannique, au Manitoba, en Ontario, au Québec, au Nouveau-Brunswick, en Nouvelle-Écosse et à Île-du-Prince-Édouard. Les tiques I. pacificus $(n=10)$ et I. angustus ( $\mathrm{n}=7$ ) ont été collectées exclusivement à Vancouver, en Colombie-Britannique. Les tiques I. scapularis ( $n=550)$ ont été collectées au Manitoba, en Ontario, au
Québec, au Nouveau-Brunswick, en Nouvelle-Écosse et à l'île-du-Prince-Édouard.

Des nymphes ont été collectées dans chacune de ces provinces, sauf au Manitoba (Winnipeg) où seul des adultes ont été prélevés (figure 2). La densité moyenne des nymphes (nymphes $/ 100 \mathrm{~m}^{2}$ [ET]), qui présentent le plus grand risque d'infection pour les humains, était la plus élevée dans les régions sentinelles de Lunenburg $(0,45[0,74])$, Montréal $(0,43[0,91])$, Granby $(0,38[0,48])$ et Kingston $(0,27[0,38])$ (tableau 1$)$. La région d'Ottawa-Gatineau a été échantillonnée en début de saison, ce qui a donné une densité plus faible de nymphes $(0,06[0,12])$, mais une densité élevée d'adultes $(0,42[0,72])$, ce qui pose également un risque important pour la santé.

\section{Analyses de laboratoire}

La prévalence d'infection à $B$. burgdorferi chez les tiques Ixodes spp. (nymphes et adultes) variait de $0 \%$ à $45 \%$ (tableau 2). La prévalence moyenne d'infection était plus élevée chez les tiques adultes $(36,3 \%)$ que chez les nymphes $(22,0 \%)$, mais cette différence $n$ 'était pas statistiquement significative (test de Wilcoxon apparié; $P=0,142 ; V=3$ ). $B$. miyamotoi a été trouvé dans deux spécimens, l'un provenant d'Ottawa-Gatineau et l'autre de Montréal. La prévalence d'infection à

A. phagocytophilum variait de $0 \%$ à $4,1 \%$ dans les régions sentinelles où des tiques $I$. scapularis ont été trouvées. $B$. microti a été détecté dans une tique $I$. angustus dans la région sentinelle de Vancouver. Une I. scapularis adulte était positive au virus Powassan dans la région sentinelle de Lunenburg.

Figure 1 : Localisation des régions sentinelles dans le Réseau sentinelle canadien pour la surveillance de la maladie de Lyme en 2019a,b

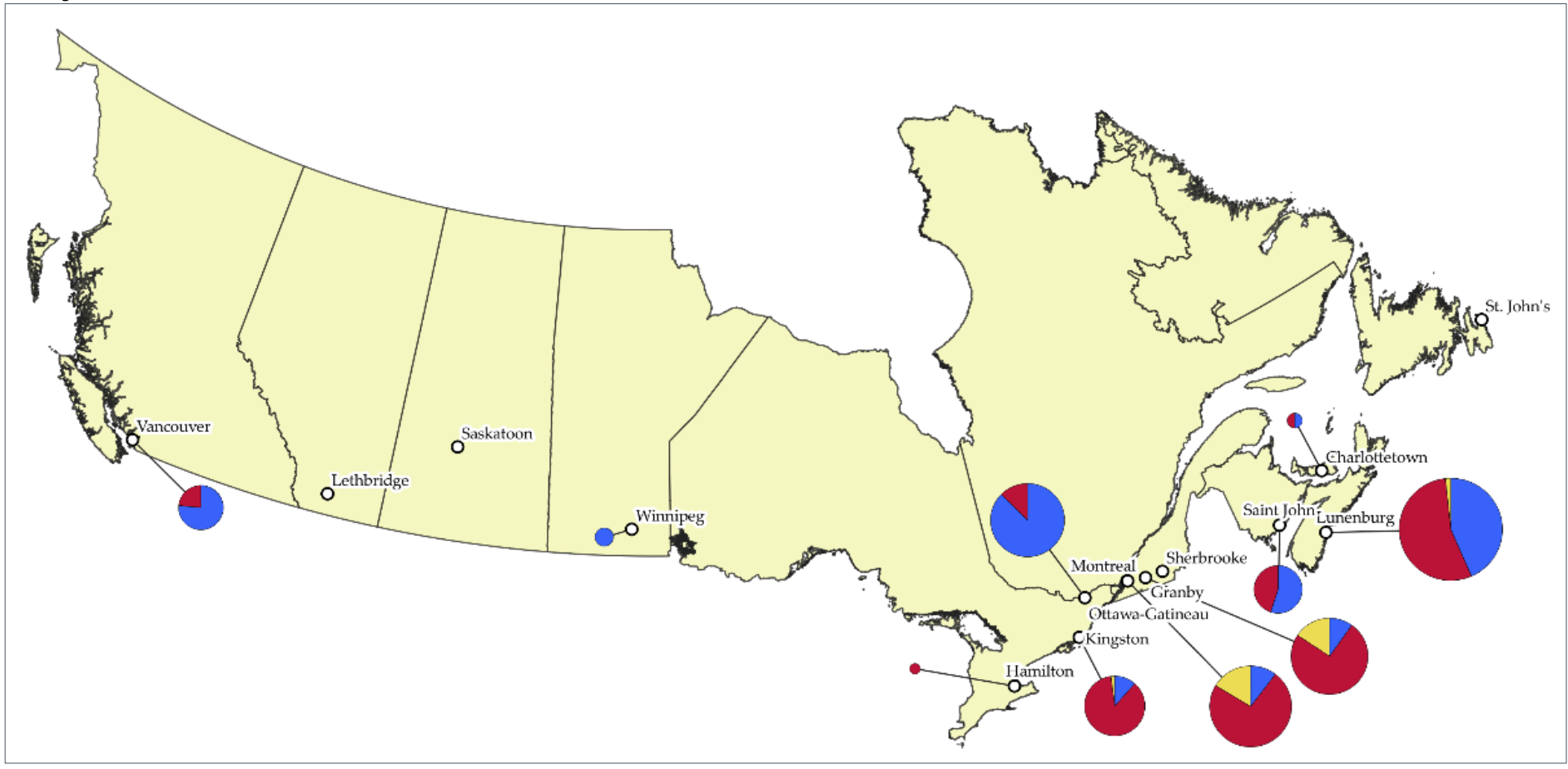

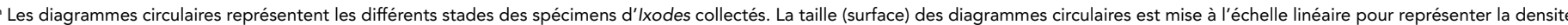
moyenne de tiques (tiques $/ 100 \mathrm{~m}^{2}$ ) dans tous les sites de surveillance de la région sentinelle Jaune (larve); rouge (nymphes); bleu (adultes) 
Figure 2 : Densités de tiques Ixodes spp. par stade (larve, nymphe et adulte) pour chaque région sentinelle du Réseau sentinelle canadien pour la surveillance de la maladie de Lyme en 2019

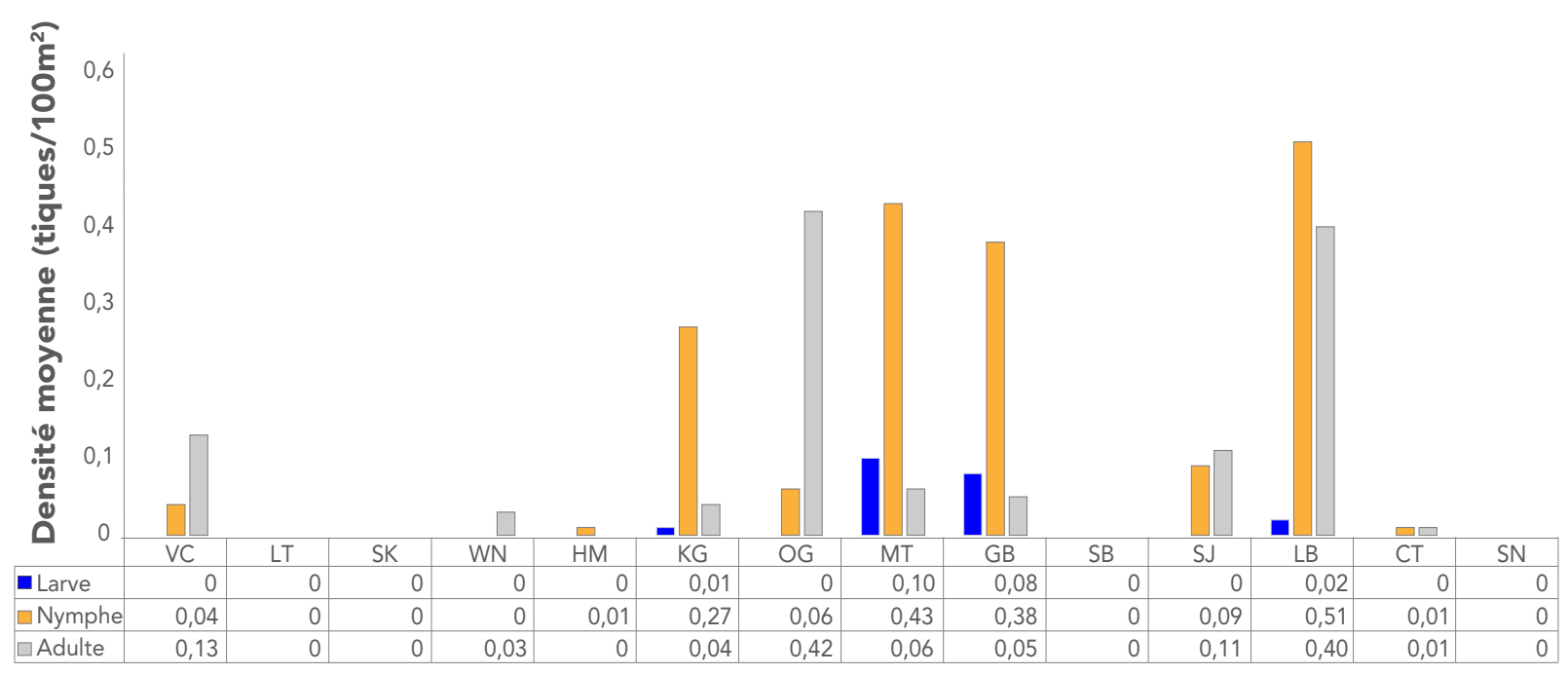

Région sentinelle

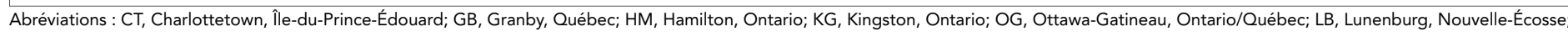

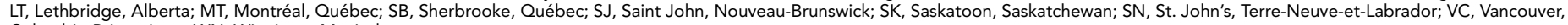
Colombie-Britannique; WN, Winnipeg, Manitoba

Tableau 2 : Abondance des tiques Ixodes spp. dans les régions sentinelles du Réseau sentinelle canadien pour la surveillance de la maladie de Lyme en 2019 et prévalence d'infection des pathogènes transmis par les tiques

\begin{tabular}{|c|c|c|c|c|c|c|c|c|c|c|c|}
\hline \multirow[t]{2}{*}{ Région sentinelle } & \multicolumn{4}{|c|}{$\begin{array}{l}\text { Abondance des espèces } d^{\prime} \text { lxodes } \\
(n)\end{array}$} & \multicolumn{7}{|c|}{ Prévalence d'infection (\%) ${ }^{a, b}$} \\
\hline & Larve & Nymphe & Adulte & Total & BbN & BbA & $\mathrm{BbT}$ & $\mathrm{Bm}$ & Ap & Bmi & POWV \\
\hline Vancouver, C.-B. & 0 & 4 & 13 & 17 & $0^{c}$ & $0^{c}$ & $0^{c}$ & $0^{c}$ & $0^{c}$ & $5,9^{e}$ & $0^{c}$ \\
\hline Lethbridge, Alb. & 0 & 0 & 0 & 0 & s.o. & s.o. & s.o. & s.o & s.o. & s.o & s.o \\
\hline Saskatoon, Sask. & 0 & 0 & 0 & 0 & s.o & s.o & s.o & s.o & s.o & s.o & s.o. \\
\hline Winnipeg, Man. & 0 & 0 & 3 & 3 & s.o & $0^{c}$ & $0^{c}$ & $0^{c}$ & $0^{c}$ & $0^{c}$ & $0^{c}$ \\
\hline Hamilton, Ont. & 0 & 2 & 0 & 2 & $0^{c}$ & $0^{c}$ & $0^{c}$ & $0^{c}$ & $0^{c}$ & $0^{c}$ & $0^{c}$ \\
\hline Kingston, Ont. & 2 & 82 & 11 & 95 & $28,0^{f}$ & $54,5^{f}$ & $31,2^{f}$ & $0^{c}$ & $1,1^{d}$ & $0^{c}$ & $0^{c}$ \\
\hline Ottawa-Gatineau, Ont./Qc & 0 & 12 & 83 & 95 & $33,3^{f}$ & $39,8^{f}$ & $38,9^{f}$ & $1,1^{\mathrm{d}}$ & $0^{c}$ & $0^{c}$ & $0^{c}$ \\
\hline Montréal, Qc & 19 & 85 & 12 & 116 & $14,1^{\mathrm{e}}$ & $66,7^{f}$ & $20,6^{f}$ & $1,0^{d}$ & $1,0^{d}$ & $0^{c}$ & $0^{c}$ \\
\hline Granby, Qc & 3 & 37 & 5 & 45 & $13,5^{\mathrm{e}}$ & $60^{f}$ & $19,0^{\mathrm{e}}$ & $0^{c}$ & $2,4^{d}$ & $0^{c}$ & $0^{c}$ \\
\hline Sherbrooke, Qc & 0 & 0 & 0 & 0 & s.o. & s.o & s.o & s.o. & s.o & s.o & s.o \\
\hline Saint John, N.-B. & 0 & 9 & 11 & 20 & $55,6^{f}$ & $36,4^{f}$ & $45^{f}$ & $0^{c}$ & $5^{e}$ & $0^{c}$ & $0^{c}$ \\
\hline Charlottetown, Î.-P.-É. & 0 & 1 & 1 & 2 & $0^{c}$ & $0^{c}$ & $0^{c}$ & $0^{c}$ & $0^{c}$ & $0^{c}$ & $0^{c}$ \\
\hline Lunenburg, N.-É. & 3 & 96 & 73 & 172 & $24,0^{f}$ & $31,5^{f}$ & $26,6^{f}$ & $0^{c}$ & $4,1^{d}$ & $0^{c}$ & $0,6^{d}$ \\
\hline St. John's, T.-N.-L. & 0 & 0 & 0 & 0 & s.o. & s.o. & s.o. & s.o. & s.o. & s.o. & s.o. \\
\hline Nombre total & 27 & 328 & 212 & 567 & s.o. & s.o. & s.o. & s.o. & s.o. & s.o. & s.o. \\
\hline Prévalence globale & s.o. & s.o. & s.o. & s.o. & 22,0 & 36,3 & 26,6 & $<0,01$ & 0,02 & $<0,01$ & $<0,01$ \\
\hline
\end{tabular}

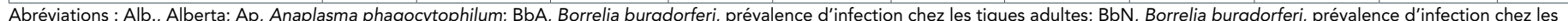

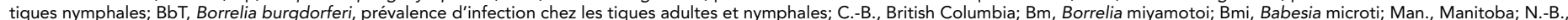

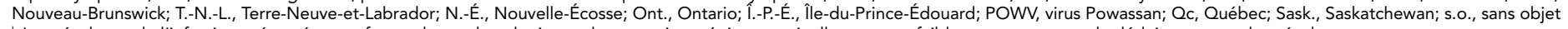

${ }^{b}$ La prévalence de l'infection présentée sous forme de nombre de tiques dans certaines régions sentinelles est trop faible pour permettre de déduire un taux de prévalence

Zéro (vert) aucune tique infectée

'Prévalence d'infection $<5 \%$ (bleu)

Prévalence d'infection entre 5 et $20 \%$ (jaune)

'Prévalence d'infection $>20 \%$ (rouge) 


\section{Discussion}

Au cours de sa première année, le Réseau sentinelle canadien pour la surveillance de la maladie de Lyme a documenté la présence de tiques Ixodes spp. qui sont vecteurs de $B$. burgdorferi et de quatre autres agents pathogènes pour les humains dans sept des dix provinces canadiennes, avec une prévalence globale d'infection de $25,2 \%$ (de $0 \%$ à $45,0 \%$ ) pour $B$. burgdorferi. Toutefois, nous avons constaté une grande variabilité entre les régions : alors qu'aucune tique positive à Borrelia n'a été trouvée en Colombie-Britannique, à l'île-du-Prince-Édouard ou au Manitoba, la prévalence d'infection dans les régions sentinelles de l'Ontario, du Québec, du Nouveau-Brunswick et de la Nouvelle-Écosse se situait entre $19,0 \%$ et $45,0 \%$. Ces résultats concordent avec les résultats d'études récentes sur la distribution des tiques $I$. scapularis au Canada (24-27), ce qui suggère que l'approche sentinelle adoptée par le ResCaL permet d'évaluer avec succès les variations régionales du risque de maladie de Lyme.

Les résultats de la surveillance ont mis en évidence une variation régionale notable de la densité d'I. scapularis en Ontario et au Québec. La densité moyenne des nymphes dans les régions de Granby et de Montréal au Québec était respectivement de 0,38 et 0,43 tique par $100 \mathrm{~m}^{2}$, alors qu'aucune tique à pattes noires n'a été trouvée à Sherbrooke. En Ontario, les densités de nymphes étaient élevées à Kingston $\left(0,27\right.$ nymphe/100 $\left.\mathrm{m}^{2}\right)$, mais beaucoup plus faibles dans le sud de l'Ontario, avec seulement 0,02 nymphe/100 $\mathrm{m}^{2}$ à Hamilton. Les densités de nymphes en Ottawa doivent être interprétées avec prudence, car l'échantillonnage a été effectué plus tôt en été, avant le pic d'activité des nymphes.

Les efforts de surveillance du ResCaL en 2019 représente les premières initiatives de surveillance active sur l'île-du-Prince-Édouard et l'île de Terre-Neuve, dans l'optique de détection des populations établis de tiques I. scapularis. Ainsi, la présence $d^{\prime} l$. scapularis confirmée par la méthode de la flanelle à l'île-du-Prince-Édouard a été une découverte inédite. La détection de deux stades différents (nymphe et adulte) dans l'environnement sur deux sites d'échantillonnage distincts est une preuve précoce de la possibilité d'une reproduction locale des tiques. Cependant, il est possible que les deux spécimens soient des tiques adventices transportées sur l'île par des oiseaux migrateurs. Une surveillance active dans le futur sera nécessaire pour confirmer si des tiques sont établies dans la province.

Les analyses en laboratoire des tiques collectées ont permis de détecter deux agents pathogènes remarquables. Tout d'abord, Babesia microti a été détectée dans une nymphe I. angustus, ce qui représente le premier signalement d'une tique infectée par cet agent pathogène en Colombie-Britannique. Deuxièmement, la lignée du virus Powassan a été détectée dans la région sentinelle de Lunenburg, ce qui n'est que la deuxième détection de cet agent pathogène dans la région (28).

\section{Forces et faiblesses}

L'un des principaux atouts de notre réseau de surveillance est la collaboration établie entre les provinces et entre les autorités de santé publique et les universitaires. Ces liens ont permis le partage des connaissances entre les parties concernées et ont été essentiels lors de la planification du réseau. De plus, ce partenariat a été primordial lors de la sélection des régions sentinelles et de la réalisation du travail sur le terrain. Pour renforcer ces collaborations, le ResCaL continuera à travailler en étroite collaboration avec les autorités sanitaires provinciales afin de s'assurer que les activités du réseau sont complémentaires et répondent aux objectifs de surveillance provinciaux.

Une limite importante à l'interprétation des résultats est le moment variable de l'échantillonnage dans chaque région. Cela peut avoir contribué aux différences dans l'abondance des stades de tiques collectées, car les adultes sont généralement actifs plus tôt au printemps alors que l'abondance des nymphes atteint son maximum un peu plus tard en été (29). Les valeurs absolues des densités de tiques rapportées doivent donc être interprétées avec prudence.

L'inclusion de variables telles que la température et les conditions météorologiques durant l'échantillonnage dans des analyses statistiques ultérieures sera également importante pour contrôler la variabilité temporelle de l'échantillonnage des tiques. Enfin, la poursuite de l'échantillonnage annuel dans un délai plus étroitement aligné avec le pic d'activité des nymphes fournira de meilleures données pour documenter l'évolution du risque régional dans le temps.

\section{Conclusion}

Le Réseau sentinelle canadien de surveillance de la maladie de Lyme représente la première initiative nationale coordonnée de surveillance active des maladies transmises par les tiques au Canada. À notre connaissance, l'approche de surveillance sentinelle n'a pas été appliquée à la maladie de Lyme à l'échelle nationale ailleurs en Amérique du Nord ou en Europe, ce qui fait du ReSCaL un modèle utile pour les autres pays touchés par la maladie de Lyme et d'autres maladies transmises par les tiques. Après l'établissement de données de base sur les vecteurs de la maladie de Lyme et la prévalence de Borrelia, la prochaine étape importante consistera à établir le lien entre le risque environnemental et l'incidence régionale des cas humains. La poursuite de la collecte de données environnementales, sociales et humaines sur les cas dans les régions sentinelles permettra d'explorer la représentativité plus large des mesures de risque basées sur les régions sentinelles pour la surveillance des maladies transmises par les tiques.

\section{Déclaration des auteurs}

C. G. - Conceptualisation, méthodologie, analyse, interprétation, rédaction originale, révision et édition P. A. L., C. B. - Conceptualisation, méthodologie, interprétation, révision et édition 
K. C., S. G., C. J., J. K., M. K., L. R. L., R. M., M. N., M. R., C. R., A. S., B. T. - Méthodologie, interprétation, révision et édition J. B., A. D., S. D., M. E., E. F., E. G., G. G., D. H., E. J., G. L., D. M., M. M., J. N., K. R., K. T., M. V. - Méthodologie

\section{Intérêts concurrents}

Aucuns.

\section{Remerciements}

Nous remercions les nombreux assistants de terrain et techniciens laboratoire qui ont participé à la collecte et à l'analyse des tiques. Nous remercions les autorités sanitaires régionales et provinciales pour leur soutien dans la sélection des sites, et les parcs régionaux et nationaux pour l'accès aux sites.

\section{Financement}

Ces travaux ont été soutenus par le Canadian Lyme Disease Research Network, un réseau financé par les Instituts de recherche en santé du Canada (IRSC). L'Institut national de santé publique du Québec (INSPQ) et le ministère de la Santé et des Services sociaux (MSSS) ont financé la collecte des données dans 12 sites d'échantillonnage des régions sentinelles du Québec dans le cadre des activités de surveillance annuelles. Le Laboratoire national de microbiologie a confirmé les déterminations des espèces et a entrepris de tester les tiques afin de détecter les pathogènes associés aux tiques pour tous les spécimens collectés dans les juridictions en dehors de l'Ontario.

\section{Références}

1. Kulkarni MA, Berrang-Ford L, Buck PA, Drebot MA, Lindsay LR, Ogden NH. Major emerging vector-borne zoonotic diseases of public health importance in Canada. Emerg Microbes Infect 2015;4(6):e33-e. DOI

2. Surveillance de la maladie de Lyme. Ottawa (ON): Gouvernement du Canada; (accédé 2020-03-02). www. canada.ca/en/public-health/services/diseases/lyme-disease/ surveillance-lyme-disease.html

3. Gabriele-Rivet V, Arsenault J, Badcock J, Cheng A, Edsall J, Goltz J, Kennedy J, Lindsay LR, Pelcat Y, Ogden NH. Different ecological niches for ticks of public health significance in Canada. PLoS One 2015 Jul;10(7):e0131282. DOI PubMed

4. Canadian Lyme Disease Research Network. 2019. Interested in our work? CLyDRN; 2019 (accédé 2020-03-02).

https://www.clydrn.ca/

5. Ogden NH, Bouchard C, Badcock J, Drebot MA, Elias SP, Hatchette TF, Koffi JK, Leighton PA, Lindsay LR, Lubelczyk CB, Peregrine AS, Smith RP, Webster D. What is the real number of Lyme disease cases in Canada? BMC Public Health 2019 Jun;19(1):849. DOI PubMed
6. Ogden $\mathrm{NH}$, Lindsay LR, Morshed M, Sockett PN, Artsob $\mathrm{H}$. The emergence of Lyme disease in Canada. CMAJ 2009 Jun;180(12):1221-4. DOI PubMed

7. Ogden NH, Maarouf A, Barker IK, Bigras-Poulin M, Lindsay LR, Morshed MG, O'callaghan CJ, Ramay F, Waltner-Toews D, Charron DF. Climate change and the potential for range expansion of the Lyme disease vector Ixodes scapularis in Canada. Int J Parasitol 2006 Jan;36(1):63-70. DOI PubMed

8. Kulkarni MA, Narula I, Slatculescu AM, Russell C. Lyme disease emergence after invasion of the blacklegged tick, lxodes scapularis, Ontario, Canada, 2010-2016. Emerg Infect Dis 2019 Feb;25(2):328-32. DOI PubMed

9. Gasmi S, Bouchard C, Ogden NH, Adam-Poupart A, Pelcat $Y$, Rees EE, Milord F, Leighton PA, Lindsay RL, Koffi JK, Thivierge K. Evidence for increasing densities and geographic ranges of tick species of public health significance other than Ixodes scapularis in Québec, Canada. PLoS One 2018 Aug;13(8):e0201924. DOI PubMed

10. Leighton P, Koffi J, Pelcat Y, Lindsay R, Ogden N. Predicting the speed of tick invasion: an empirical model of range expansion for the Lyme disease vector Ixodes scapularis in Canada. J Appl Ecol 2012;49(2):457-64. DOI

11. Aenishaenslin C, Michel P, Ravel A, Gern L, Milord F, Waaub JP, Bélanger $D$. Factors associated with preventive behaviors regarding Lyme disease in Canada and Switzerland: a comparative study. BMC Public Health 2015 Feb;15(1):185. DOI PubMed

12. Ripoche M, Gasmi S, Adam-Poupart A, Koffi JK, Lindsay LR, Ludwig A, Milord F, Ogden NH, Thivierge K, Leighton PA. Passive tick surveillance provides an accurate early signal of emerging Lyme disease risk and human cases in southern Canada. J Med Entomol 2018 Jun;55(4):1016-26. DOI PubMed

13. Koffi JK, Leighton PA, Pelcat Y, Trudel L, Lindsay LR, Milord F, Ogden NH. Passive surveillance for I. scapularis ticks: enhanced analysis for early detection of emerging Lyme disease risk. J Med Entomol 2012 Mar;49(2):400-9. DOl PubMed

14. Frazão TD, Camilo DG, Cabral EL, Souza RP. Multicriteria decision analysis (MCDA) in health care: a systematic review of the main characteristics and methodological steps. BMC Med Inform Decis Mak 2018 Nov;18(1):90. DOl PubMed

15. Ogden NH, Radojevic M, Wu X, Duvvuri VR, Leighton PA, Wu J. Estimated effects of projected climate change on the basic reproductive number of the Lyme disease vector Ixodes scapularis. Environ Health Perspect 2014 Jun;122(6):631-8. DOI PubMed

16. Diuk-Wasser MA, Hoen AG, Cislo P, Brinkerhoff R, Hamer SA, Rowland M, Cortinas R, Vourc'h G, Melton F, Hickling GJ, Tsao JI, Bunikis J, Barbour AG, Kitron U, Piesman J, Fish D. Human risk of infection with Borrelia burgdorferi, the Lyme disease agent, in eastern United States. Am J Trop Med Hyg 2012 Feb;86(2):320-7. DOI PubMed

17. Clow KM, Finer R, Lumsden G, Jardine CM. Assessing the repeatability of tick dragging as a method for Ixodes scapularis surveillance. Vector Borne Zoonotic Dis 2018 Nov;18(11):628-31. DOl PubMed 
18. Stafford KC III, Cartter ML, Magnarelli LA, Ertel SH, Mshar PA. Temporal correlations between tick abundance and prevalence of ticks infected with Borrelia burgdorferi and increasing incidence of Lyme disease. J Clin Microbiol 1998 May;36(5):1240-4. DOI PubMed

19. Mather TN, Nicholson MC, Donnelly EF, Matyas BT. Entomologic index for human risk of Lyme disease. Am J Epidemiol 1996 Dec;144(11):1066-9. DOl PubMed

20. Pepin KM, Eisen RJ, Mead PS, Piesman J, Fish D, Hoen AG, Barbour AG, Hamer S, Diuk-Wasser MA. Geographic variation in the relationship between human Lyme disease incidence and density of infected host-seeking Ixodes scapularis nymphs in the Eastern United States. Am J Trop Med Hyg 2012 Jun;86(6):1062-71. DOI PubMed

21. Schillberg E, Lunny D, Lindsay LR, Nelder MP, Russell C, Mackie M, Coats D, Berry A, Young Hoon KN. Distribution of Ixodes scapularis in northwestern Ontario: Results from active and passive surveillance activities in the Northwestern Health Unit Catchment Area. Int J Environ Res Public Health 2018 Oct;15(10):2225. DOI PubMed

22. Tokarz R, Tagliafierro T, Cucura DM, Rochlin I, Sameroff S, Lipkin WI. Detection of Anaplasma phagocytophilum, Babesia microti, Borrelia burgdorferi, Borrelia miyamotoi, and Powassan virus in ticks by a multiplex real-time reverse transcription-PCR assay. MSphere 2017 Apr;2(2):e00151-17. DOI PubMed

23. R Core Team. R: A language and environment for statistical computing. Vienna (AT): R Foundation for Statistical Computing; 2019 (accédé 2020-03-02). https://www.R-project.org/
24. Gasmi S, Ogden NH, Ripoche M, Leighton PA, Lindsay RL, Nelder MP, Rees E, Bouchard C, Vrbova L, Rusk R, Russell C, Pelcat Y, Mechai S, Kotchi SO, Koffi JK. Detection of municipalities at-risk of Lyme disease using passive surveillance of Ixodes scapularis as an early signal: A province-specific indicator in Canada. PLoS One 2019 Feb;14(2):e0212637. DOI PubMed

25. Villeneuve A, Goring J, Marcotte L, Overvelde S. Seroprevalence of Borrelia burgdorferi, Anaplasma phagocytophilum, Ehrlichia canis, and Dirofilaria immitis among dogs in Canada. Can Vet $J$ 2011 May;52(5):527-30. PubMed

26. Ouhoummane N, Adam-Poupart A, Milord F, Irace-Cima A, Thivierge K, Therrien C. Rapport de surveillance de la maladie de Lyme : année 2016. Quebec (QC) : Institut national de santé publique du Québec; 2017. https://www.inspq.qc.ca/ publications $/ 2296$

27. Clow KM, Ogden NH, Lindsay LR, Michel P, Pearl DL, Jardine CM. Distribution of ticks and the risk of Lyme disease and other tick-borne pathogens of public health significance in Ontario, Canada. Vector Borne Zoonotic Dis 2016 Apr;16(4):215-22. DOI PubMed

28. Tick borne diseases response plan: May 2020. Halifax (NS): Province of Nova Scotia; 2020-05-12 (accédé 202008-27). https://novascotia.ca/dhw/cdpc/documents/ Tick-Borne-Disease-Response-Plan.pdf

29. Rodgers SE, Miller NJ, Mather TN. Seasonal variation in nymphal blacklegged tick abundance in southern New England forests. J Med Entomol 2007 Sep;44(5):898-900. DOI PubMed 


\section{Appendice : Liste d'affiliations}

1 Département de pathologie et microbiologie, et Groupe de recherche en épidémiologie des zoonoses et santé publique (GREZOSP), Faculté de médecine vétérinaire, Université de Montréal, Montréal, $\mathrm{QC}$

${ }^{2}$ Faculté de médecine et des sciences de la santé, Université de Sherbrooke, Sherbrooke, OC

${ }^{3}$ Office of the Chief Medical Officer of Health, New Brunswick Department of Health, Fredericton, NB

${ }^{4}$ Department of Pathobiology, Ontario Veterinary College, University of Guelph, Guelph, ON

${ }^{5}$ Nova Scotia Health Authority, Halifax, NS

${ }^{6}$ Agriculture and Agri-Food Canada, Lethbridge Research and Development Centre, Lethbridge, $A B$

${ }^{7}$ Laboratoire national de microbiologie, Agence de la santé publique du Canada, Winnipeg, MB

${ }^{8}$ Department of Companion Animals, Atlantic Veterinary College, University of Prince Edward Island, Charlottetown, PEI

${ }^{9}$ Communicable Diseases \& Immunization Service, British Columbia Centre for Disease Control, Victoria, BC

${ }^{10}$ School of Population and Public Health, Faculty of Medicine, University of British Columbia, Vancouver, BC

${ }^{11}$ Zoonotic Diseases and Emerging Pathogens, British Columbia Centre for Disease Control, Vancouver, BC

12 Division d'intégration des politiques et des zoonoses, Centre des maladies infectieuses d'origine alimentaire, environnementale et zoonotique, Agence de la santé publique du Canada, SaintHyacinthe, $\mathrm{QC}$
${ }^{13}$ PEI Provincial Microbiology Laboratory, Health PEI, Charlottetown, PEI

${ }^{14}$ Department of Health and Community Services, Public Health Division, St. John's, NL

${ }^{15}$ University of Saskatchewan, Saskatoon, SK

${ }^{16}$ School of Epidemiology and Public Health, University of Ottawa, Ottawa, ON

${ }^{17}$ KFL\&A Public Health, Kingston, ON

${ }^{18}$ Faculty of Forestry \& Environmental Management, University of New Brunswick, Fredericton, NB

${ }^{19}$ Enteric, Zoonotic and Vector-Borne Diseases, Communicable Diseases, Emergency Preparedness and Response, Public Health Ontario, Toronto, ON

${ }^{20}$ Surveillance, évaluation de risque et contrôle des maladies infectieuses, Institut national de santé publique du Québec, Montréal, QC

${ }^{21}$ Department of Entomology, University of Manitoba, Winnipeg, MB

${ }^{22}$ Laboratoire de santé publique du Québec, Saint-Anne-deBellevue, QC

${ }^{23}$ Division des sciences des risques pour la santé publique, Laboratoire national de microbiologie, Agence de la santé publique du Canada, Saint-Hyacinthe, QC 